\title{
Actin disruption agents induce phosphorylation of histone H2AX in human breast adenocarcinoma MCF-7 cells
}

\author{
IK JAE SHIN ${ }^{1}$, YONG-TAE AHN ${ }^{3}$, YONGKUK KIM ${ }^{4}$, JONG-MYOUNG KIM ${ }^{5}$ and WON G. AN ${ }^{1,2}$ \\ ${ }^{1}$ Joint Research Center of Pusan National University-Fraunhofer IGB; ${ }^{2}$ School of Korean Medicine, Busan 609-735; \\ ${ }^{3}$ Department of Biomedical Laboratory Science, Kyungdong University, San 91-1 Bongpo-ri, Toseong-myeon, \\ Goseong-gun, Gangwon-do 219-832; ${ }^{4}$ Laboratory of Mathematical and Computational Biology, \\ Department of Mathematics, Kyungpook National University, Daegu 702-701; ${ }^{5}$ Department of Marine \\ BioMaterials and Aquaculture, Pukyong National University, Busan 608-737, Republic of Korea
}

Received November 5, 2010; Accepted December 15, 2010

DOI: $10.3892 /$ or.2011.1214

\begin{abstract}
Modified actin dynamics are a unique feature of transformed cancer cells and thereby promising targets for cancer chemotherapy. While latrunculin B (LB) and pectenotoxin-2 (PTX-2), both derived from natural sources, inhibit actin polymerization, jasplakinolide (JSP) prevents actin depolymerization. The purpose of this study was to examine the detailed molecular action of actin disruption inducing apoptosis via double strand breaks (DSBs). Actin disruption induced phosphorylation of H2AX, a well known DSB marker leading to $\mathrm{G} 2$ arrest and consequently resulted in apoptosis on MCF-7 cancer cells. Cells impaired by actin disruption activated Erk (extracellular signal-related kinase) and p53 protein was involved in DNA damage responses, but did not change the levels of $\mathrm{p} 21^{\mathrm{Cip} 1 / \mathrm{WAF} 1}$ protein in MCF-7 cells. To overcome the DSBs by actin disruption, MCF-7 cells set the repair system through the homologous recombination (HR) pathway. These results indicate that actin is involved in the signaling inducing DSBs and HR repair as well as G2 cell cycle arrest in human cancer. Therefore, the results suggest that actin disruption might be a potential candidate for developing anti-cancer therapies in human breast cancer.
\end{abstract}

Correspondence to: Dr Won G. An, School of Korean Medicine, Busan 609-735, Republic of Korea

E-mail:wgan@pusan.ac.kr

Abbreviations: ATM, ataxia telangiectasia mutated; BCA, bicinchoninic acid; BRCA1, breast cancer associated protein 1; DSB, double strand break; Erk, extracellular signal-related kinase; JSP, jasplakinolide; HR, homologous recombination; LB, latrunculin B; NHEJ, non-homologous end-joining; PTX-2, pectenotoxin-2; PIKKs, phosphatidylinositol-3 kinase-like kinases

Key words: actin cytoskeleton, latrunculin B, DNA double strand break, BRCA1, G2/M, p53, pectenotoxin-2

\section{Introduction}

Cells have to struggle to protect their genomic information from numerous environmental stresses during their lifetime. Among the genomic damage within the cell, DNA double-strand break (DSB) is probably the most dangerous. As soon as DSB is generated, histone $\mathrm{H} 2 \mathrm{AX}$ is rapidly phosphorylated on serine 139 residues from the carboxylterminus to form $\gamma \mathrm{H} 2 \mathrm{AX}$ at the incipient sites of DSB by the phosphatidylinositol-3 kinase-like kinases (PIKKs) (1). One of the PIKKs, ataxia telangiectasia mutated (ATM) is a primary signal transducer in response to DSB and mediates the downstream signal proteins such as Chk1/2, p53, breast cancer associated protein 1 (BRCA1), and extracellular signal-related kinase $(2,3)$. Serving as binding sites for repair proteins and chromatin remodeling complexes, $\gamma \mathrm{H} 2 \mathrm{AX}$ plays an important role for the response to conserve genomic stability $(4,5)$. Cells have to operate two repairing system, homologous recombination and non-homologous end-joining (NHEJ), to renovate their damaged DSB. Two pathways are distinguished from the accuracy of repair and the requirement of homologous sequence, sister chromatid. HR is an error-free repair system but limiting to replicating cells in the $\mathrm{S}, \mathrm{G} 2 / \mathrm{M}$ phase of the cell cycle. In contrast, NHEJ is an error-prone system in all cell cycles of eukaryotes (6). HR is carried out by RAD52 epistasis group, which is implicated with a large number of proteins such as BRCA1, BRCA2, XRCC2 and XRCC3. BRCA1, a target of ATM, associated with BRCA2 and RAD51 forms complex which is scrutinizing the genome on the intact homologous sister chromatid and repairing the missing information on the broken strand without loss of genetic information. In NHEJ, Ku heterodimer binds to the DNA DSB ends and then DNA-PKcs forms a complex with Ku/DNA strands. This protects damaged DNA strands from nuclease digestion prior to ligation. Subsequently, the compromised DNA double stands are directly ligated together by the DNA ligase IV/XRCC4 complex (7). However, the precise mechanism of DSB repair is not completely elucidated and the components of these two systems are controversial (8). 
As highly conserved during the evolution, actin is the most abundant and ubiquitous protein in eukaryotic cells. Actin dynamics in response to external and internal stimuli are responsible for cellular processes such as cellular membrane reorganization, motility, and cytokinesis. Previous studies revealed that the nuclear actin is involved in chromatin remodeling, mRNA transcription and transcription factor activity (9-11). Cancer cells generally were characterized by distinct changes e.g. alteration of nuclear/cytoplasmic ratio, alteration of cell adhesion, increase of motility and invasion, and disruption of cell division and apoptosis machineries. Since these unique morphological and phenotypical changes are known to be involved in the alteration of actin dynamics, understanding the mechanism of actin dynamics in cancer cells may allow a precise strategy to be formed $(12,13)$.

Natural products disrupting the actin dynamics are thought to be potent anti-cancer agents, and thereby postulated to control cancer growth and proliferation. Actin polymerization is inhibited by swinholide A, latrunculin B (LB) and pectenotoxin-2 (PTX-2), in contrast to actin stabilizers, such as jasplakinolide (JSP) and phalloidin, disrupting actin depolymerization (14).

We searched the detailed molecular action of actin disruption on human breast cancer MCF-7 cells. Cancer cells treated with actin disrupting agents show apoptotic results including DSB, delaying the cell cycle at G2 and activation of p53 as well as ERK. The results indicate that actin disrupting agents could be a potential candidate for developing anti-cancer therapy in the human breast cancer cells.

\section{Materials and methods}

Cell culture, reagents and antibodies. Human breast cancer (MCF-7) cells were obtained from American Type Culture Collection (ATCC). Cells were grown in Dulbecco's modified Eagle's medium supplemented with $1 \%$ antibiotic-antimycotic (Gibco) and 10\% fetal bovine serum (Gibco), and incubated at $37^{\circ} \mathrm{C}$ in a humidified atmosphere of $5 \% \mathrm{CO}_{2}$. In order to address the cells to signals required for survival from actin disrupting agents, all experiment on DSB induction were carried out without serum and exogenous growth factors. Chemical reagents and antibodies were from following suppliers: latrunculin B (LB), jasplakinolide (JSP), anti-mouse p53 and BRCA1 monoclonal antibody from Calbiochem; pectenotoxin-2 (PTX-2) as a gift from Dr J.H. Jung, Pusan National University, Korea (15); propodium iodide, RNase A from Sigma; protease inhibitor cocktail tablets from Roche; nitrocellulose transfer membrane from Whatman; bicinchoninic acid (BCA) protein assay kit from Pierce (USA); CellTiter 96 Non-Radioactive Cell Proliferation Assay kit for MTT assay kit from Promega (USA); enhanced chemiluminescence (ECL) detection kit, IgG-specific monoclonal, IgG-polyclonal and FITC-monoclonal antibodies form GE Healthcare; anti-rabbit PARP, phosphor-Erk polyclonal antibodies from Cell Signaling Technology; anti-mouse phosphor-H2AX antibody (Ser139, clone JBW301) from Upstate; anti-mouse p21 ${ }^{\mathrm{WAF} / \mathrm{CIF} 1}$ antibody from BD transduction laboratories, anti-mouse DNA-PK antibody from Santa Cruz Biotechnology.
Viability assay. Cells/well $\left(2 \times 10^{4}\right)$ were incubated in 96-well flat bottom plates in $100 \mu \mathrm{l}$ of medium and stabilized in a humidified atmosphere of $5 \% \mathrm{CO}_{2}$ at $37^{\circ} \mathrm{C}$ for more than $12 \mathrm{~h}$. Cells were exposed to $\mathrm{LB}(0.5,1.5,3.0 \mu \mathrm{M})$ and maintained for $24 \mathrm{~h}$. The MTT assay was performed by manufacturer's instruction. The absorbance at $570 \mathrm{~nm}$ was determined using a multi plate reader (Perkin-Elmer, Model 1420 Victor $3^{\mathrm{TM}}$ ). Triplicate wells were assayed for each condition, the mean and standard deviations were determined. Mean \pm SE were calculated and reported as the percentage of proliferation compared to control.

Immunocytochemistry. MCF-7 cells were grown on coverslip that were rinsed with cold PBS. For permeabilization, cells were incubated with $150 \mu 1$ permeabilizing buffer (PB; $0.15 \mathrm{M}$ $\mathrm{NaCl}, 10 \mathrm{mM}$ Tris, $1 \mathrm{mM} \mathrm{MgCl}{ }_{2}, 0.2 \mathrm{mM}$ dithiothreitol (DTT), $0.5 \mathrm{mM} \mathrm{CaCl}_{2}, 25 \%$ glycerol, pH 8.0) (16) for 4-5 min; $150 \mu \mathrm{l}$ PB $+0.5 \%$ Triton X-100 for $3.5 \mathrm{~min} ; 150 \mu \mathrm{l}$ PB for $30 \mathrm{~min}$ at $37^{\circ} \mathrm{C}$ and washed with PBS-T. Cells were fixed in $4 \%$ paraformaldehyde (pH 7.2) for $15 \mathrm{~min}$ and washed twice with PBS-T (PBS + 0.2\% Tween-20). Before cell staining, cells were blocked in PBS-T-S (PBS-T $+10 \%$ FBS) for 20 min. All antibodies were diluted in PBS-T-S. Cells were detected with primary DNA-PK (1:100), BRCA1 (1:100) for $1 \mathrm{~h}$ and washed with PBS-T. FITC-mouse second antibody (1:200) was incubated with rhodamine-phalloidin (1:500) and DAPI $(0.5 \mathrm{mg} / \mathrm{ml})$ for $60 \mathrm{~min}$ in the dark. After washing with PBS-T, the cells were observed under a fluorescent microscope (Olympus IX-71, Japan) and a confocal microscope (Olympus IX-81, Japan).

Cell extraction. Harvested cells were lysed in a total lysis buffer [protease inhibitor cocktail 1 tablet $/ 50 \mathrm{ml}$ TNES buffer (50 mM Tris-HCl, pH 7.4, 1\% NP-40, 2 mM EDTA, $100 \mathrm{mM}$ $\mathrm{NaCl})]$ on ice for $30 \mathrm{~min}$. After centrifugation for $10 \mathrm{~min}$ at $12,000 \mathrm{rpm}$, the supernatant was stored at $-70^{\circ} \mathrm{C}$ until its use. Acid extraction of histones was performed as follows. Cells were washed with PBS and resuspended in NETN buffer $(150 \mathrm{mM} \mathrm{NaCl}, 1 \mathrm{mM}$ EDTA pH 8.0, $20 \mathrm{mM}$ Tris- $\mathrm{HCl}$ $\mathrm{pH} 8.0,0.5 \% \mathrm{NP}-40$ ). After 10-min incubation on ice, nuclei were pelleted by centrifugation at $8,500 \mathrm{rpm}$ for $5 \mathrm{~min}$ at $4^{\circ} \mathrm{C}$. The pellet was resuspended in $0.1 \mathrm{M} \mathrm{HCl}$ and incubated for $10 \mathrm{~min}$ at room temperature. Acid-extracted histones were obtained by centrifugation at $8,500 \mathrm{rpm}$ for $5 \mathrm{~min}$ at $4^{\circ} \mathrm{C}$.

Western blot analysis. The protein concentration was determined by bicinchoninic acid (BCA, Pierce) protein assay according to the manufacturer's instructions. Total proteins $(30 \mu \mathrm{g})$ were resolved by 8 or $12 \%$ SDS-PAGE gels, and then electrophoretically transferred onto a nitrocellulose transfer membrane (Whatman) by electroblotting. The membranes were soaked in TBS-T buffer [ $20 \mathrm{mM}$ Tris, $\mathrm{pH} 7.4 ; 137 \mathrm{mM}$ $\mathrm{NaCl} ; 0.1 \%$ (v/v) Tween-20] containing 5\% (w/v) non-fat milk, and were probed with the specific primary antibodies diluted in TBS-T. The proteins were then detected with the respective secondary antibody-linked horseradish peroxidase followed by enhancement of chemiluminescence (ECL, GE Healthcare). Ponceau-S (Sigma) staining of membranes and anti-actin was applied to confirm equal loading of the proteins and successful transfer to the membranes. 


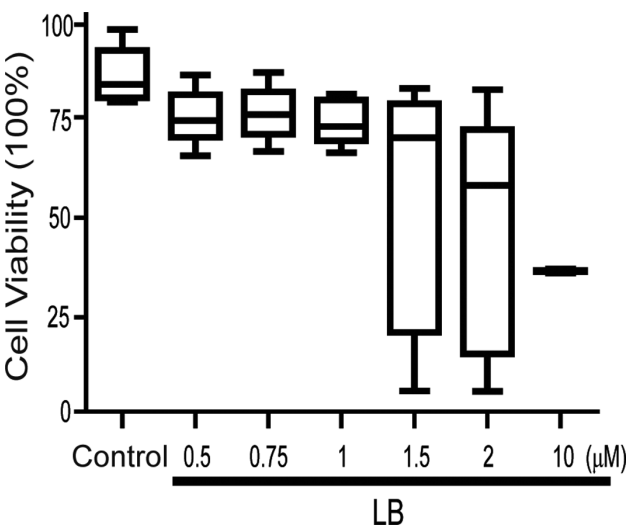

Figure 1. Effect of LB on the viability of MCF-7 cells. MCF-7 cells were treated with different concentrations of LB for $24 \mathrm{~h}$, thereafter cell viability was determined by MTT assay. The values are represented as the percentage cell inhibition where untreated control cells were regarded as $100 \%$ (mean $\pm \mathrm{SE}, \mathrm{n}=12$ ).

Flow cytometry analysis for cell cycle and protein expression. For flow cytometry analysis, cells were treated with various concentrations of LB in a 6 well-plate in a humidified atmosphere of $5 \% \mathrm{CO}_{2}$ at $37^{\circ} \mathrm{C}$ for $6-24 \mathrm{~h}$. Cells were washed with ice-cold PBS, and fixed in $100 \%$ ethanol at $-20^{\circ} \mathrm{C}$ for up to 2 weeks before the analysis of DNA contents or the expression level of $\gamma \mathrm{H} 2 \mathrm{AX}$, DNA-PKcs and BRCA1. To analyze the cell cycle, cells were stained with propidium iodide (PI) solution containing $50 \mu \mathrm{g} / \mathrm{ml} \mathrm{PI}, 0.1 \%$ nonidet P-40 (NP-40), $0.1 \%$ sodium citrate, and $100 \mu \mathrm{l} / \mathrm{ml}$ RNase $\mathrm{A}$ in the dark at $37^{\circ} \mathrm{C}$ for $30 \mathrm{~min}$. To address LB-mediated protein expression, cells were stained with anti-phospho-histone H2AX (1:500), anti-DNA-PKcs (1:500) and anti-BRCA1 (1:500) antibodies at $37^{\circ} \mathrm{C}$ for $1 \mathrm{~h}$. Cells were incubated with secondary antibody (FITC-anti-mouse 1:500) for $1 \mathrm{~h}$ at room temperature. The percent of cells in each stage of the cell cycle and $\gamma \mathrm{H} 2 \mathrm{AX}$, DNA-PKcs and BRCA1 protein expression were analyzed with FACSCalibur flow cytometry (BD Bioscience, France).

\section{Results}

$L B$ induces cell growth inhibition in MCF-7 cells. The cytotoxicity of actin disrupting agent is a crucial obstacle in developing anticancer drugs based on actin. To designate a proper concentration of LB, the MTT assay was performed on MCF-7 breast cancer cells (Fig. 1). The viability of MCF-7 cells decreased in a dose-dependent manner upon treatment with various concentrations of LB $(0.5-10 \mu \mathrm{M})$ for $24 \mathrm{~h}$. The cell viability decreased rapidly from LB treatment at the concentration higher than $1.0 \mu \mathrm{M}$. The $\mathrm{IC}_{50}$ of $\mathrm{LB}$ was $7.1 \mu \mathrm{M}$ for $24 \mathrm{~h}$ in MCF-7 cells. After being incubated with 1.5 and $10 \mu \mathrm{M}$ LB for $24 \mathrm{~h}$, cell viability was decreased by $\sim 65.6$ and $41.5 \%$ compared to that of control group, respectively.

$L B$ destabilizes actin microfilaments and induces apoptosis in MCF-7 cells. In order to confirm the effect of LB on the morphology of human breast cancer cells, morphological feature and fluorescent image were examined by fluorescent and confocal microscopy. After $24 \mathrm{~h}$ of incubation with $1.5 \mu \mathrm{M}$ of LB in MCF-7 cells, the morphological change of



Figure 2. Disruption of actin microfilaments and induction of apoptosis by LB in MCF-7 cells. MCF-7 cells were treated with or without LB $(1.5 \mu \mathrm{M})$ for $24 \mathrm{~h}$. Phase contrast image (A) was taken by phase contrast and fluorescent microscope (Olympus IX-71, Japan). Cells were fixed with $4 \%$ paraformaldehyde and stained DNA with DAPI (B) or immunostained F-actin with rhodamine-phalloidine (C) using confocal microscope (Olympus IX-81, Japan). Each panel is representative of at least three experiments $(\times 800)$.

the cells was immediately investigated by phase contrast microscopy. Cell shape rapidly rounded with subcortical membrane following treatment with LB, although they maintained attachment to the plate. MCF-7 cells treated with LB gradually showed prominent morphological changes such as cytoplasmic shrinkage and marked convolution of cellular surface for $24 \mathrm{~h}$ (Fig. 2A). MCF-7 cells were exposed to $1.5 \mu \mathrm{M}$ of $\mathrm{LB}$ for $24 \mathrm{~h}$, and then assessed for morphological signs of apoptosis by staining with DAPI and for F-actin with rhodamine-phalloidin, then monitored by confocal microscopy. A distinguishing feature of apoptosis is the condensation and fragmentation of nuclear chromatin. Nuclear condensation and apoptotic bodies were observed in cells treated with LB (Fig. 2B). F-actin staining images showed that untreated control MCF-7 cells possessed a well-developed actin cytoskeleton with numerous subcortical actin filaments and stress fibers, while LB treated cells displayed slightly decreased F-actin and irregular aggregates of phalloidinreactive material appeared in confocal microscopy images (Fig. 2C). These results elucidated that LB induced apoptosis through actin disruption in MCF-7 cells.

$L B$ induces apoptosis in a time- and dose-dependent manner. In order to determine whether LB treatment induces apoptosis, the level of proteins involved in apoptotic signal transduction was examined by Western blotting. MCF-7 cells were treated with different concentrations of LB $(0.1$, 1.5 and $3 \mu \mathrm{M}$ ) for $24 \mathrm{~h}$ and with $1.5 \mu \mathrm{M}$ of LB for 6,12 or $24 \mathrm{~h}$ ) (Fig. 3A and B). PARP cleavage was monitored with PARP antibodies by Western blot analysis, which specifically recognizes the 89-kDa fragment of the cleaved PARP and the uncleaved 116-kDa PARP. As shown in Fig. 3, the proportion of uncleaved 116-kDa PARP was decreased and the part of the 89-kDa cleavage product was increased with the treatment of LB in a time- and dose-dependent manner. However, cleaved 89-kDa PARP fragment except for the intact 116-kDa protein was not detected in the control cells (Fig. 3, lane 1). As shown 

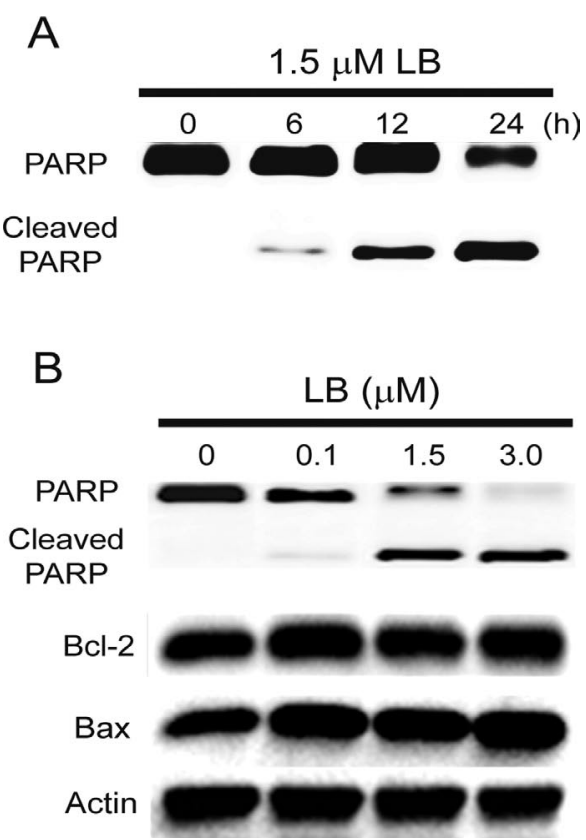

Figure 3. Effect of LB on the induction of PARP cleavage. MCF-7 cells were treated with $1.5 \mu \mathrm{M} \mathrm{LB}$ for 6,12 and $24 \mathrm{~h}(\mathrm{~A})$ and $\mathrm{LB}(0.1,1.5$ and $3.0 \mu \mathrm{M})$ for $24 \mathrm{~h}$ (B). Protein $(30 \mu \mathrm{g})$ from cell total lysates was electrophoresed on SDS-PAGE, transferred to nitrocellulose, and probed with PARP, anti-Bcl-2, and anti-Bax antibodies respectively. Anti-actin was used for ensuring equal amount of protein loading.

in Fig. 3B, the level of Bax was slightly increased but Bcl-2 protein showed no change upon LB treatment in either cell line. The ratio of proapoptotic Bax and antiapoptotic $\mathrm{Bcl}-2$ is known to be a barometer of apoptosis (17). Apoptotic signals activate Bax protein to reduce Bcl-2 in mitochondria, which is releasing the cytochrome $\mathrm{c}$ from mitochondria, supporting that actin disruption causes apoptosis through Bax signaling in MCF-7 cells.
$L B$ induces double-strand breaks (DSB) in a time- and dose-dependent manner. DSB resulting from destructive DNA damage recruits phosphorylated $\mathrm{H} 2 \mathrm{AX}$ in a broad damaged region at the early stage. Detection of $\gamma \mathrm{H} 2 \mathrm{AX}$ level is probably the most authentic method developed to date for measuring the DSB in cells. To investigate whether the apoptosis by LB is involved in DNA damage, especially for DSB, the level of $\gamma \mathrm{H} 2 \mathrm{AX}$ was measured by Western blotting and flow cytometry. As shown in Fig. 4, actin disruption introduced $\gamma \mathrm{H} 2 \mathrm{AX}$ to the DSB regions increasingly in a time- and dose-dependent manner in human cancer cells. The population of FITC-conjugated second antibody binding to $\gamma \mathrm{H} 2 \mathrm{AX}$ showed a time-dependent increase at $1.5 \mu \mathrm{M}$ concentration of $\operatorname{LB}(13.3 \%, 12 \mathrm{~h}$ to $15.2 \%, 24 \mathrm{~h})$ and dose-dependently in MCF-7 cells (Fig. 4A). The result was confirmed by Western blot analysis (Fig. 4B and C). Other actin disrupting agents, $0.25 \mu \mathrm{M}$ JSP and $1 \mu \mathrm{M}$ PTX-2, also recruited the $\gamma \mathrm{H} 2 \mathrm{AX}$ protein to protect from DNA damage (Fig. 4D). The results indicate that actin disruption induces DSB time- and dosedependently in MCF-7 cells.

Actin disruption by $L B$ arrests the cell cycle at G2 phase due to DNA damage. DSB is a severe genotoxic lesion as it leads to defective genomic integrity and genomic instability. Therefore, it is necessary to arrest the cell cycle to repair the lesions and help to maintain genomic stability. To assess whether LB induced DSB also prevents cells from transiting the cell cycle, MCF-7 cells treated with $1.5 \mu \mathrm{M} \mathrm{LB}$ for 12,18 and $24 \mathrm{~h}$ were subjected to FACS analysis. The ratio of G2/M phase cells treated with $1.5 \mu \mathrm{M} \mathrm{LB}$ was $65.4 \%$ compared to $15.4 \%$ in the controls (Fig. 5A). The amount of phosphorylated Erk, one of the effectors of DNA damage gradually increased as the concentration of LB increased for $24 \mathrm{~h}$ in MCF-7 cancer cells (Fig. 5B). In response to a variety of stress damaging DNA including DSB, p53, a well known tumor suppressor, protects mammalian cells from tumor by inducing cell cycle

\section{A}



B

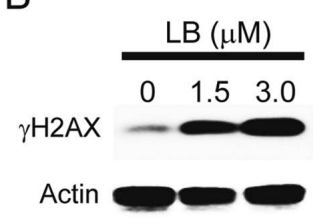

C



JSP $(\mu \mathrm{M}) \quad 0 \quad 0.25 \quad 0$

PTX-2 ( $\mu \mathrm{M}) \quad 0 \quad 0 \quad 1$

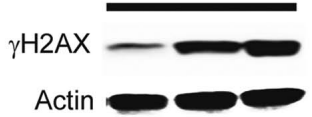

Figure 4. Effect of LB on the induction of DSB in MCF-7 cells. The $\gamma \mathrm{H} 2 \mathrm{AX}$ expression of MCF-7 cells treated with designated concentrations of LB and time were analyzed by flow cytometry (A) and Western blotting (B and C). The protein level of $\gamma \mathrm{H} 2 \mathrm{AX}$ in MCF-7 cells treated with other actin disrupting agent, JSP $0.25 \mu \mathrm{M}$ or PTX-2 $1 \mu \mathrm{M}$ for $24 \mathrm{~h}$ is shown by Western blotting (D). Protein $(5 \mu \mathrm{g})$ from cell acid lysates was electrophoresed on SDS-PAGE, transferred to nitrocellulose, and probed with $\gamma \mathrm{H} 2 \mathrm{AX}$ antibodies. Anti-actin was used to ensure equal amount of protein loading. 
A
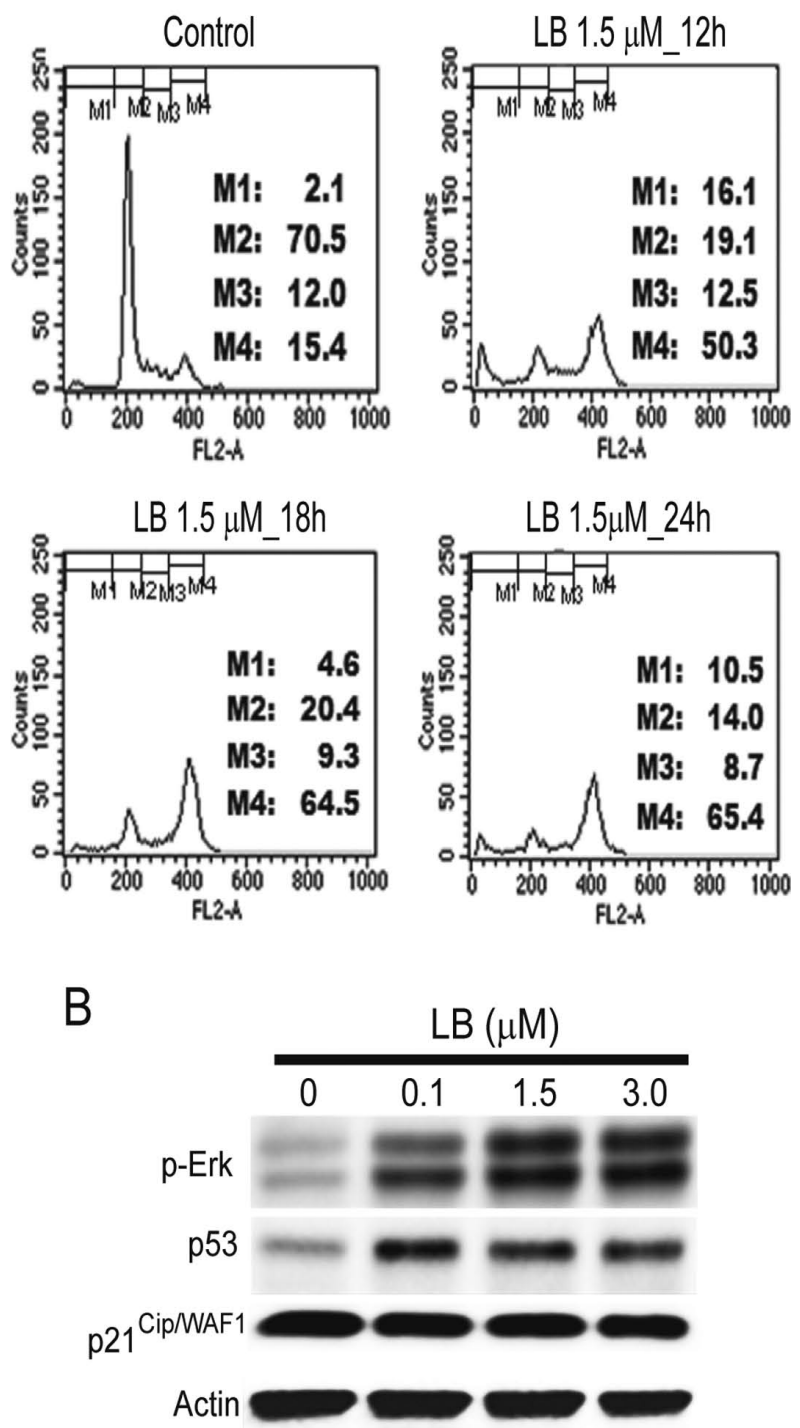

Figure 5. Effect of LB on the cell cycle progression and signal transduction in MCF-7 cells. (A) The cell cycle of MCF-7 treated with $1.5 \mu \mathrm{M}$ LB for 12,18 and $24 \mathrm{~h}$ were analyzed by flow cytometry. Control indicates the cells subjected to the same treatment without LB. DNA was stained with PI and the contents of DNA were determined by flow cytometry. Numbers indicate the percentage of cells in the phase of cell cycle (M1, G0; M2, G1; M3, S; M4, G2). (B) The signal transduction of MCF-7 treated with various concentrations of $\mathrm{LB}(0.5,1.5$ and $1.5 \mu \mathrm{M})$ was analyzed by Western blotting. Protein $(30 \mu \mathrm{g})$ from cell total lysates was electrophoresed on SDS-PAGE, transferred to nitrocellulose, and probed with phospho-Erk, anti-p53, p21 Cip1/WAF1 $^{2}$ antibodies, respectively. Anti-actin was used to ensure equal amount of protein loading.

arrest, DNA repair and apoptosis. p53 plays a pivotal role in $\mathrm{G} 2$ cell cycle arrest and DSB repair $(18,19)$. As shown in Fig. $4 \mathrm{~B}, \mathrm{p} 53$ protein was stabilized by $\mathrm{LB}$ treatment for $24 \mathrm{~h}$ in MCF-7 cells. However, LB did not affect the level of p21 $1^{\mathrm{Cip} 1 /}$ WAF1, a p53 downstream effector and cdk inhibitor, in MCF-7 cancer cells (Fig. 4B). We also investigated the pathway of DSB repair evoked by $\mathrm{LB}$.

DSB by actin disruption is repaired through the HR pathway. In response to DSB, cells activate the component protein of HR or NHEJ pathway to repair their DNA. In order to
A

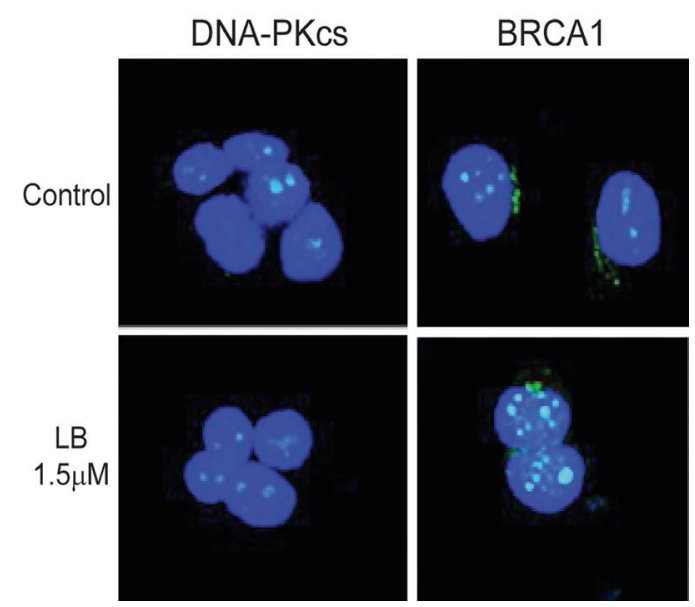

Figure 6. DSB by actin disruption was repaired through HR pathway in MCF-7 cells. MCF-7 cells treated without and with $1.5 \mu \mathrm{M}$ for $24 \mathrm{~h}$ were immunostained with DNA-PKes (A) and BRCA1 (B) primary antibody followed by secondary antibody conjugated with FITC. Samples were photographed using confocal microscopy at $\times 400$.

investigate which pathway is predominant by actin disruption, the expression level of DNA-PKcs for NHEJ and BRCA1 for HR was examined by confocal image analysis. MCF-7 cells were incubated with $1.5 \mu \mathrm{M} \mathrm{LB}$ for $24 \mathrm{~h}$ and washed with cold PBS. Upon permeabilizing and fixing treatment, cells were immunostained with primary antobodies against DNA-PKcs and BRCA1 followed by secondary antibody conjugated with FITC. As shown in Fig. 6A and B, treatment of MCF-7 cells with $\mathrm{LB}$ activated BRCA1 protein expression to repair DNA damage. The expression of BRCA1 in the nucleus was particularly prominent in the cells permeabilized before fixation, indicating that DSB by actin disruption was repaired through HR pathway. Therefore, the results suggested that LB induces $\mathrm{G} 2$ cell cycle arrest to provide time for repairing DSB by HR pathway.

\section{Discussion}

The prominent features of cancer cells include their viability in any circumstance and immortality due to endless cell cycle. These abnormal growth properties of cancer cells are associated with modified actin and a large number of actin-related proteins. Since modified actin dynamics are responsible to tumorigenesis, it is important to understand the mechanism of actin dynamics in cancer for developing a novel anticancer drug (20). Induction of apoptosis has been recognized as a good strategy for the identification of anti-cancer drugs. There have been a number of studies targeting actin cytoskeleton involved in apoptosis in eukaryotic cells including cancer cell, although the effect of actin disruption in apoptosis induction in cancer cells is controversial (21-24). One of the important initiation elements in the apoptotic pathway is the activation of caspases followed by cleavage of PARP, the main substrates of caspase. This is in accordance with our observation of apoptotic bodies and PARP cleaved by LB (Fig. 3).

Of all DNA damage types, DSB is particularly dangerous and induce apoptosis. It has been found that actin disrupting 
agents induced phosphorylated $\mathrm{H} 2 \mathrm{AX}$ against DSB and arrested cell cycle at G2 phase in MCF-7 breast cancer cells (Figs. 4 and 5). Since phosphorylation of H2AX is considered as a specific marker of DSB induction (25), our data indicate that the phosphorylated $\mathrm{H} 2 \mathrm{AX}$ is recruited to their impaired DNA by LB at time- and dose-dependently in human cancer cells. It is known that p53 accumulation plays an important role in the repair and cell cycle arrest (26). p53, downstream molecule of ATM, was understood as a major component of the DNA damage response pathway. After the introduction of DNA injuries the level of p53 protein rises, which in turn induces a transient cell cycle arrest or apoptotic cell death (27). It has been assumed that LB-mediated DSB recruited $\gamma \mathrm{H} 2 \mathrm{AX}$ around the DSB regions and then stabilized p53 via ATM-dependent pathway. The contribution of p53 to G2 phase cell cycle arrest involves some of its transcriptional targets such as $\mathrm{p} 21^{\mathrm{WAF} 1 / \mathrm{CIF} 1}$, GADD45, and 14-3-3s (28). $\mathrm{p} 21^{\mathrm{WAF} 1 / \mathrm{CIF} 1}$ is known to be involved in G2 arrest as well as G1 cell cycle arrest. This protein is accumulated in nucleus at the initiating step of mitosis and interacts with cyclin B1 at the G2 phase $(29,30)$. As shown in Fig. 5, p21 WAF//CIF1 is not involved in G2 phase arrest by actin disruption. Therefore, it can be anticipated that DSB induced by LB is implicated with GADD45 or 14-3-3s, which is a downstream signals of p53. The novelty of the present findings is that histone $\mathrm{H} 2 \mathrm{AX}$ also is phosphorylated in cells by LB at the G2 boundary. Previous studies demonstrated that actin cytoskeleton plays a central role in early mitosis along with microtubules in eukaryotic cells (31-33). Erk1/2, a key player of the cell cycle belonging to one of the MAPK family, regulate the cell cycle and the progress of apoptosis after DNA damage (6,34). In vitro, actin disrupted by latrunculin B or cytochalasin D arrests the cell cycle at G2 phase in yeast or human cells (35). It is suggested that the expression of Erk1/2 was activated by DNA damage and involved in regulating actin disruption in order to control the beginning of mitosis (Fig. 5).

To maintain genetic stability, cells have developed precise mechanisms to repair DSB implicated with the cell cycle. It has been previously suggested that the sister chromatid, present in the late S, G2/M phases of the cell cycle, would favor conservative HR during these phases of the cell cycle, which indicates that HR has a role in DSB repair in S/G2 phase (36). Error prone NHEJ pathway is the simplest way to cure DSB triggered by DNA-PKcs forming juxtaposition with the Ku70/80 heterodimer. BRCA1 may bring about functional interaction with BRCA2, which mediates critically RAD51 strand transferase in HR error-free pathway. Although the exact role of DNA-PKcs and BRCA1 are not clear in DSB repair, actin disruption-mediated DSB may be repaired through HR pathway in that the activation of BRCA1 is higher than that of DNA-PKcs in G2 phase arrest. Although BRCA1 is known to be a mammalian regulator of two DSB repair pathway, this protein mainly controls HR repair in G2 phase $(37,38)$. Considering the results of $\mathrm{G} 2$ arrest and BRCA1 expression (Figs. 5 and 6), LB mediated-DSB is repaired through HR pathway in cells.

In summary, actin cytoskeleton is involved in apoptosis through DSB and HR repairing system. DSB responses then activated Erk and p53 which mediated DSB repair and G2 cell cycle arrest. This is the first report of DSB, the most detrimental of DNA damage, induced by actin disruption. Based on our findings and studies of other investigators, we propose a model showing the relationship of actin disruption, DNA damage, DNA damage responses, Erk and p53 in the induction of growth inhibition and apoptosis of cancer cells.

\section{Acknowledgements}

This study was supported by 2008 PNU-IGB Joint Research Center Grant of Pusan National University. J.M.K. was supported by Grant \#20088033 from the MLTM, Korea.

\section{References}

1. Rogakou EP, Pilch DR, Orr AH, Ivanova VS and Bonner WM: DNA double-stranded breaks induce histone H2AX phosphorylation on serine 139. J Biol Chem 273: 5858-5868, 1998.

2. Lavin MF and Kozlov S: ATM activation and DNA damage response. Cell Cycle 6: 931-942, 2007.

3. Tang D, Wu D, Hirao A, et al: ERK activation mediates cell cycle arrest and apoptosis after DNA damage independently of p53. J Biol Chem 277: 12710-12717, 2002.

4. Bonner WM, Redon CE, Dickey JS, et al: GammaH2AX and cancer. Nat Rev Cancer 8: 957-967, 2008.

5. Huang J, Liang B, Qiu J and Laurent BC: ATP-dependent chromatin-remodeling complexes in DNA double-strand break repair: remodeling, pairing and (re)pairing. Cell Cycle 4: 1713-1715, 2005.

6. Mao Z, Bozzella M, Seluanov A and Gorbunova V: DNA repair by non-homologous end joining and homologous recombination during cell cycle in human cells. Cell Cycle 7: 2902-2906, 2008.

7. Jackson SP: Sensing and repairing DNA double-strand breaks. Carcinogenesis 23: 687-696, 2002.

8. Shrivastav M, De Haro LP and Nickoloff JA: Regulation of DNA double-strand break repair pathway choice. Cell Res 18: 134-147, 2008.

9. Miralles $\mathrm{F}$ and Visa $\mathrm{N}$ : Actin in transcription and transcription regulation. Curr Opin Cell Biol 18: 261-266, 2006.

10. Farrants AK: Chromatin remodelling and actin organisation. FEBS Lett 582: 2041-2050, 2008.

11. Vartiainen MK, Guettler S, Larijani B and Treisman R: Nuclear actin regulates dynamic subcellular localization and activity of the SRF cofactor MAL. Science 316: 1749-1752, 2007.

12. Giganti A and Friederich E: The actin cytoskeleton as a therapeutic target: state of the art and future directions. Prog Cell Cycle Res 5: 511-525, 2003.

13. Rao J and Li N: Microfilament actin remodeling as a potential target for cancer drug development. Curr Cancer Drug Targets 4: 345-354, 2004.

14. Allingham JS, Klenchin VA and Rayment I: Actin-targeting natural products: structures, properties and mechanisms of action. Cell Mol Life Sci 63: 2119-2134, 2006.

15. Jung JH, Sim CJ and Lee CO: Cytotoxic compounds from a two-sponge association. J Nat Prod 58: 1722-1726, 1995.

16. Okorokov AL, Rubbi CP, Metcalfe S and Milner J: The interaction of p53 with the nuclear matrix is mediated by F-actin and modulated by DNA damage. Oncogene 21: 356-367, 2002.

17. Agarwal MK, Agarwal ML, Athar M and Gupta S: Tocotrienolrich fraction of palm oil activates $\mathrm{p} 53$, modulates $\mathrm{Bax} / \mathrm{Bcl} 2$ ratio and induces apoptosis independent of cell cycle association. Cell Cycle 3: 205-211, 2004.

18. Bunz F, Dutriaux A, Lengauer C, et al: Requirement for $\mathrm{p} 53$ and p21 to sustain G2 arrest after DNA damage. Science 282: 1497-1501, 1998.

19. Houtgraaf JH, Versmissen J and van der Giessen WJ: A concise review of DNA damage checkpoints and repair in mammalian cells. Cardiovasc Revasc Med 7: 165-172, 2006.

20. Jordan MA and Wilson L: Microtubules and actin filaments: dynamic targets for cancer chemotherapy. Curr Opin Cell Biol 10: 123-130, 1998.

21. Martin SS and Leder P: Human MCF10A mammary epithelial cells undergo apoptosis following actin depolymerization that is independent of attachment and rescued by Bcl-2. Mol Cell Biol 21: 6529-6536, 2001. 
22. Chae HD, Choi TS, Kim BM, Jung JH, Bang YJ and Shin DY: Oocyte-based screening of cytokinesis inhibitors and identification of pectenotoxin-2 that induces Bim/Bax-mediated apoptosis in p53-deficient tumors. Oncogene 24: 4813-4819, 2005.

23. Gu LZ, Hu WY, Antic N, Mehta R, Turner JR and de Lanerolle P: Inhibiting myosin light chain kinase retards the growth of mammary and prostate cancer cells. Eur J Cancer 42: 948-957, 2006.

24. Ahmed SA, Odde S, Daga PR, et al: Latrunculin with a highly oxidized thiazolidinone ring: structure assignment and actin docking. Org Lett 9: 4773-4776, 2007.

25. Sedelnikova OA and Bonner WM: GammaH2AX in cancer cells: a potential biomarker for cancer diagnostics, prediction and recurrence. Cell Cycle 5: 2909-2913, 2006.

26. Gatz SA and Wiesmuller L: p53 in recombination and repair. Cell Death Differ 13: 1003-1016, 2006.

27. Foijer F and te Riele H: Check, double check: the G2 barrier to cancer. Cell Cycle 5: 831-836, 2006.

28. Taylor WR and Stark GR: Regulation of the G2/M transition by p53. Oncogene 20: 1803-1815, 2001

29. Gillis LD, Leidal AM, Hill R and Lee PW: p21Cip1/WAF1 mediates cyclin B1 degradation in response to DNA damage. Cell Cycle 8: 253-256, 2009.

30. Takizawa CG and Morgan DO: Control of mitosis by changes in the subcellular location of cyclin-B1-Cdk1 and Cdc25C. Curr Opin Cell Biol 12: 658-665, 2000.
31. Hwang E, Kusch J, Barral Y and Huffaker TC: Spindle orientation in Saccharomyces cerevisiae depends on the transport of microtubule ends along polarized actin cables. J Cell Biol 161: 483-488, 2003.

32. Hyman AA and White JG: Determination of cell division axes in the early embryogenesis of Caenorhabditis elegans. J Cell Biol 105: 2123-2135, 1987.

33. McCartney BM, McEwen DG, Grevengoed E, Maddox P, Bejsovec A and Peifer M: Drosophila APC2 and Armadillo participate in tethering mitotic spindles to cortical actin. Nat Cell Biol 3: 933-938, 2001.

34. Chambard JC, Lefloch R, Pouyssegur J and Lenormand P: ERK implication in cell cycle regulation. Biochim Biophys Acta 1773: 1299-1310, 2007.

35. Gachet Y, Tournier S, Millar JB and Hyams JS: A MAP kinasedependent actin checkpoint ensures proper spindle orientation in fission yeast. Nature 412: 352-355, 2001.

36. Hendrickson EA: Cell-cycle regulation of mammalian DNA double-strand-break repair. Am J Hum Genet 61: 795-800, 1997.

37. Durant ST and Nickoloff JA: Good timing in the cell cycle for precise DNA repair by BRCA1. Cell Cycle 4: 1216-1222, 2005.

38. Moynahan ME, Chiu JW, Koller BH and Jasin M: Brcal controls homology-directed DNA repair. Mol Cell 4: 511-518, 1999. 